\title{
Produção e caracterização de espumas de alumina pelo processo gelcasting sem controle atmosférico
}

\section{(Production and characterization of alumina foams by the gelcasting process without atmospheric contro)}

\author{
E. de Sousa ${ }^{1}$, F. S. Ortega ${ }^{2}$, V. C.Pandolfelli ${ }^{1}$ \\ ${ }^{1}$ Departamento de Engenharia de Materiais - DEMa, Universidade Federal de S. Carlos \\ UFSCar, S. Carlos, SP 13565-905 \\ ${ }^{2}$ Instituto de Pesquisa e Desenvolvimento - IP\&D, Universidade do Vale do Paraíba \\ UNIVAP, S. José dos Campos, SP 12244-000 \\ eliandra.sousa@gmail.com,vicpando@power.ufscar.br,fortega@univap.br
}

\begin{abstract}
Resumo
O processo de gelcasting, associado à aeração de suspensões cerâmicas, permite a produção de espumas cerâmicas com uma ampla faixa de porosidade (50-90vol.\%). Uma das etapas críticas do processo é o enrijecimento da espuma, o qual se baseia na gelificação por meio da polimerização in situ de monômeros previamente adicionados à suspensão cerâmica. Sabidamente, tal reação é inibida pela presença de oxigênio, o que tornou usual o controle atmosférico durante a produção e gelificação da espuma. Neste trabalho, estudou-se a viabilidade de produzir espumas de alumina pelo processo de gelcasting sem controle atmosférico. Para a produção das espumas, dois diferentes agentes espumantes foram testados. As propriedades físicas e mecânicas das espumas cerâmicas, bem como a microestrutura, foram avaliadas e comparadas com resultados obtidos em condições idênticas, porém com controle atmosférico. Os resultados mostraram que ambos os processos proporcionam características semelhantes e com potencial de aplicação tecnológica.
\end{abstract}

Palavras-chave: espumas cerâmicas, alumina, processo gelcasting.

\begin{abstract}
The gelcasting process with the aeration of ceramic suspensions allows the production of ceramic foams with a wide range of porosity (50-90vol.\%.). One of the critical steps of the process is the setting of the foam, which is based on a gelling reaction by the in-situ polymerization of monomers previously added in the ceramic suspension. This reaction is inhibited by the presence of oxygen, which requires atmospheric control for the stabilization of the foam. In this work, the feasibility of producing alumina foams by the gelcasting process without atmospheric control was studied. For the foams production, two different foaming agents were tested. The physical and mechanical properties of ceramic foams, as well the microstructure, were evaluated and compared with those produced with the same foaming agents but with atmospheric control. The results obtained using the two procedures were similar and with potential to be applied with technological purposes.
\end{abstract}

Keywords: ceramic foams, alumina, gelcasting process.

\section{INTRODUÇÃO}

Cerâmicas celulares são materiais frágeis de elevada porosidade que apresentam na sua estrutura poros fechados, abertos ou interconectados [1]. A grande variedade de aplicações para as cerâmicas celulares tem motivado o desenvolvimento de diversos processos de fabricação para estes materiais, dentre os quais se destacam: controle das condições de sinterização com o objetivo de alcançar uma parcial densificação [2], incorporação de materiais orgânicos ao pó cerâmico, que se volatilizam durante a queima [3], e a réplica de espumas poliméricas $[4,5]$. Em geral, estes processos proporcionam corpos com baixa resistência mecânica a verde, microestruturas com grande quantidade de trincas, ou ainda poros com extremidades agudas que atuam como concentradores de tensão mecânica. 
Estas características limitam a resistência mecânica do produto, impondo sérias restrições ao seu uso em aplicações estruturais. Portanto a fabricação de cerâmicas celulares com propriedades mecânicas superiores apresenta um grande potencial a ser ainda explorado.

Nos últimos anos, várias técnicas foram desenvolvidas com o intuito de permitir a conformação fluida de peças cerâmicas densas com geometria complexa e de conferir ao compacto verde maior resistência mecânica, de modo que possa ser manipulado com facilidade ou até mesmo submetido a usinagem [6]. Algumas destas técnicas são baseadas na formação de um gel físico e/ou químico, após a conformação da suspensão cerâmica, convertendo-a de um líquido viscoso em um sólido elástico. Dentre estas, o processo gelcasting [7], por apresentar características únicas como baixa viscosidade da suspensão, cura rápida e alta resistência mecânica a verde, pôde ser associado a agentes espumantes e técnicas de aeração, dando origem a uma nova categoria de cerâmicas celulares [8]. Neste processo, a gelificação é obtida por meio da polimerização in situ de monômeros solúveis em água, formando uma rede de cadeias ramificadas e com ligações cruzadas que causam uma drástica mudança nas propriedades viscoelásticas da suspensão aerada à medida que a reação se desenvolve, culminando em um corpo celular rígido gelificado. Entre as características das espumas cerâmicas produzidas por este método, destacam-se elevada porosidade (até $90 \%$ ), células com geometria tendendo à esfericidade e tamanho na faixa de 10-2000 $\mu \mathrm{m}$, e paredes das células densas, proporcionando um significativo aumento na resistência mecânica específica destes materiais [9]. No entanto, alguns aspectos restringiam a maior aceitação deste processo, como por exemplo o uso de monômeros considerados moderadamente ou totalmente tóxicos e a necessidade de manter a atmosfera inerte durante a produção da espuma cerâmica. Recentemente, estudos mostraram a identificação de monômeros de baixa toxicidade (acrilato de amônio, hidroximetilacrilamida, metacrilamida e metilobisacrilamida) adequados à produção de espumas cerâmicas por meio do processo gelcasting [10, 11] e viabilidade do uso de sistemas gelificantes naturais termorreversíveis [12], ou seja, que dispensam a necessidade de manter a atmosfera inerte durante a produção da espuma cerâmica, além de serem não-tóxicos e de baixo custo.

O presente trabalho tem como objetivo estudar a possibilidade de produzir espumas de alumina pelo processo gelcasting sem controle atmosférico, bem como as condições necessárias para que a gelificação ocorra ao ar. Adicionalmente, serão comparadas as propriedades físicas, mecânicas e a microestrutura das espumas obtidas com aquelas de espumas produzidas com controle atmosférico.

\section{MATERIAIS E MÉTODOS}

Suspensões cerâmicas com teor de sólidos de 35 e $40 \mathrm{vol} . \%$ foram preparadas por meio da dispersão da alumina calcinada (Alcoa, A-1000 SG, $\mathrm{d}_{50}=0,3 \mu \mathrm{m}$ ) em solução aquosa contendo 30vol.\% de monômeros orgânicos,
$\mathrm{N}, \mathrm{N}, \mathrm{N}$ ',N'-hidroximetilacrilamida (HMAM), metacrilamida (MAM), metilenobisacrilamida (MBAM), Sigma-Aldrich, em proporção molar 3:3:1. A suspensão foi dispersa com poliacrilato de amônio (Dispersal 130, Lubrizol) e desaglomerada em moinho de bolas por $15 \mathrm{~min}$. Em seguida, a suspensão foi dividida em alíquotas de $100 \mathrm{~g}$, às quais foram adicionadas diferentes concentrações $(0,4,0,5$ e $0,6 \%$ em relação à massa de suspensão) de agente espumante nãoaniônico (Fongraminox KC-B, Clariant, ou Lutensol ON110, BASF). Esta mistura foi agitada em batedeira doméstica por aproximadamente $3 \mathrm{~min}$. Em seguida, quantidades prédeterminadas de catalisador (tetrametiletilenodiamina TEMED, Sigma) e iniciador (persulfato de amônio - APS, Aldrich) foram adicionadas para promover a gelificação do sistema. Após rápida homogeneização, a espuma foi vertida em béquers de $600 \mathrm{~mL}$, onde ocorreu a gelificação. Os corpos-de-prova foram desmoldados após permanecer em estufa a $55^{\circ} \mathrm{C}$ durante $24 \mathrm{~h}$. Após esta etapa, com auxílio de uma serra-copo foram cortados cilindros com $19 \mathrm{~mm}$ de diâmetro. Posteriormente, as amostras foram queimadas ao ar, com uma taxa de aquecimento inicial de $5{ }^{\circ} \mathrm{C} / \mathrm{min}$ até $200{ }^{\circ} \mathrm{C}$, prosseguindo com uma taxa de $1{ }^{\circ} \mathrm{C} / \mathrm{min}$ até atingir $500{ }^{\circ} \mathrm{C}$, na qual permaneceu por $1 \mathrm{~h}$ para a eliminação do material orgânico. Por fim, aplicou-se uma taxa de $5{ }^{\circ} \mathrm{C} /$ min até a temperatura de sinterização, $1600{ }^{\circ} \mathrm{C}$, na qual permaneceu por $3 \mathrm{~h}$. O resfriamento seguiu-se inercialmente até a temperatura ambiente.

A densidade geométrica $\left(\rho_{\text {geo }}\right)$ foi determinada a partir das dimensões e massa dos corpos-de-prova. A fração de vazios das espumas de alumina foi determinada pela porosidade $\varepsilon$, expressa por $\left[1-\left(\rho_{\text {geo }} / \rho_{t}\right)\right]$, considerando-se $\rho_{t}$ $=3,98 \mathrm{~g} / \mathrm{cm}^{3}$. A morfologia e a microestrutura das espumas foram analisadas por microscopia eletrônica de varredura (MEV, Philips, XL-30).

A resistência mecânica foi avaliada por ensaio de compressão uniaxial utilizando-se corpos cilíndricos com $14 \mathrm{~mm}$ de diâmetro, em uma máquina de ensaios universal (Instron 4301) à temperatura ambiente. Esses ensaios foram realizados com velocidade de carregamento de 0,5 $\mathrm{mm} / \mathrm{min}$ e demais condições de teste de acordo com norma ASTM C496-90. As superfícies das amostras ortogonais a aplicação de carga de compressão foram cobertas por uma borracha semi-rígida, para eliminar os efeitos do carregamento localizado, devido as variações na topografia das amostras. Os dados experimentais de resistência mecânica à compressão foram ajustados pela equação $\mathrm{A}$, proposta por Gibson- Ashby (GA) [13].

$$
\frac{\sigma_{0}}{\sigma_{s}}=C_{1}\left(\frac{\rho_{0}}{\rho_{s}}\right)^{n}
$$

onde $\sigma_{\mathrm{o}}$ é a resistência do material celular, $\sigma_{\mathrm{s}}$ é resistência da fração sólida, $\rho_{\mathrm{o}} / \rho_{\mathrm{S}}$ é a densidade relativa ( $\rho_{\mathrm{o}}$ é a densidade do material celular e $\rho_{\mathrm{s}}$ é a densidade da fração sólida), $\mathrm{C}_{1}$ é uma constante relacionada com a forma da célula e o expoente $n$ é uma constante igual a 1,5 para uma estrutura 
constituída por células cúbicas. Todo o procedimento de caracterização mecânica foi realizado conforme publicado em trabalhos anteriores [14].

\section{RESULTADOS E DISCUSSÃO}

No processo de gelcasting a gelificação do sistema em solução aquosa ocorre por meio da copolimerização via radical livre de monômeros vinílicos e divinícos, a qual envolve três etapas: iniciação, propagação e terminação. Essa reação não ocorre espontaneamente a temperatura ambiente e requer a adição de algum iniciador, freqüentemente um par redox. O sistema persulfato de amônio (APS) e tetrametiletilenodiamina (TEMED), respectivamente iniciador $\mathrm{e}$ catalisador, tem sido um dos mais usados para a produção de géis de poliacrilamida, poliacrilato e polimetacrilato [15]. O fato de a reação ocorrer de maneira idêntica em toda a extensão do corpo resulta em géis com excelente homogeneidade. No entanto, as etapas de iniciação e propagação têm sua cinética de reação diminuída na presença de oxigênio, devido à alta reatividade dos radicas livres com este elemento. Para que a polimerização ocorresse dentro de um intervalo de tempo compatível com o processo, elevadas concentrações dos reagentes químicos (iniciador e catalisador) foram utilizadas: $1,5 \%$ de APS em relação à massa da supensão cerâmica, associado ao TEMED em razão molar de 1:1 [16].

A Fig. 1 mostra o efeito da concentração dos agentes espumantes (Fongraminox e Lutensol) sobre o volume de espuma gerado e da densidade geométrica dos corpos de alumina após a sinterização. Em ambos os casos, o volume de espuma aumenta com o aumento da concentração do agente espumante, decorrente da redução da energia interfacial líquido-gás. Com o uso do Fongraminox foi possível obter um volume maior de espuma, enquanto que para o Lutensol o volume de espuma permaneceu quase constante ( $\cong 180 \mathrm{~mL}$ ). O alto poder espumante do Fongraminox pode

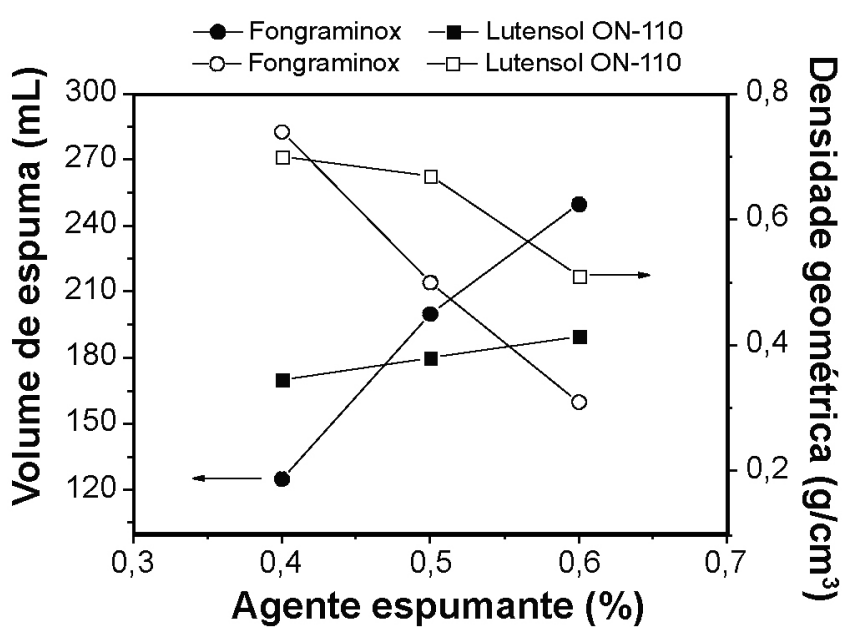

Figura 1: Efeito da concentração do agente espumante sobre o volume de espuma e densidade geométrica dos corpos de alumina após a sinterização.

[Figure 1: Effect of the concentration of foaming agent on the foam volume and geometric density alumina foams after sintering.] estar relacionado à presença de dois grupos polares em uma molécula relativamente pequena, o que proporciona um alto balanço hidrofílico-hidrofóbico (HLB), característica essa associada a um maior poder espumante. Como esperado, a densidade dos corpos de alumina após a sinterização apresentou-se inversamente proporcional à concentração de agente espumante. Os corpos de alumina preparados com Lutensol apresentaram uma estreita faixa de densidade geométrica $\left(0,51-0,70 \mathrm{~g} / \mathrm{cm}^{3}\right)$, enquanto que os preparados com Fongraminox apresentaram uma faixa de densidade geométrica mais ampla, $0,31-0,74 \mathrm{~g} / \mathrm{cm}^{3}$.

A porosidade dos corpos de alumina em função concentração dos agentes espumantes com e sem controle atmosférico, é mostrada na Fig. 2. Em ambos os casos é possível observar que, à medida que a concentração do agente espumante aumenta, a porosidade das amostras também aumenta. A porosidade das cerâmicas preparadas com Fongraminox sem controle atmosférico variou na faixa de $81-92 \%$, enquanto que aquelas com Lutensol variaram
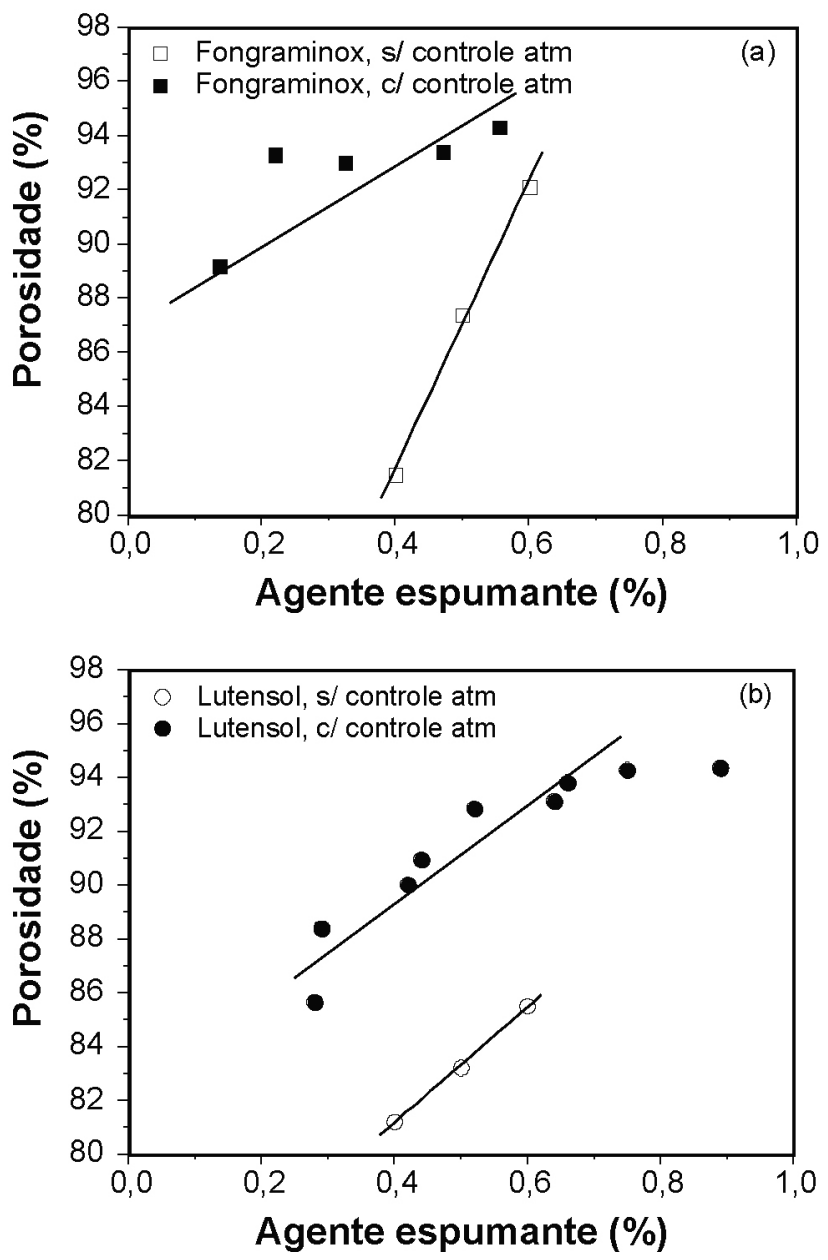

Figura 2: Porosidade das espumas de alumina produzidas com e sem controle atmosférico em função da concentração do agente espumante: a) Fongraminox e b) Lutensol.

[Figure 2: Porosity of alumina foams produced either with of without atmospheric control as a function of concentration of foaming agent: a) Fongraminox and b) Lutensol.] 

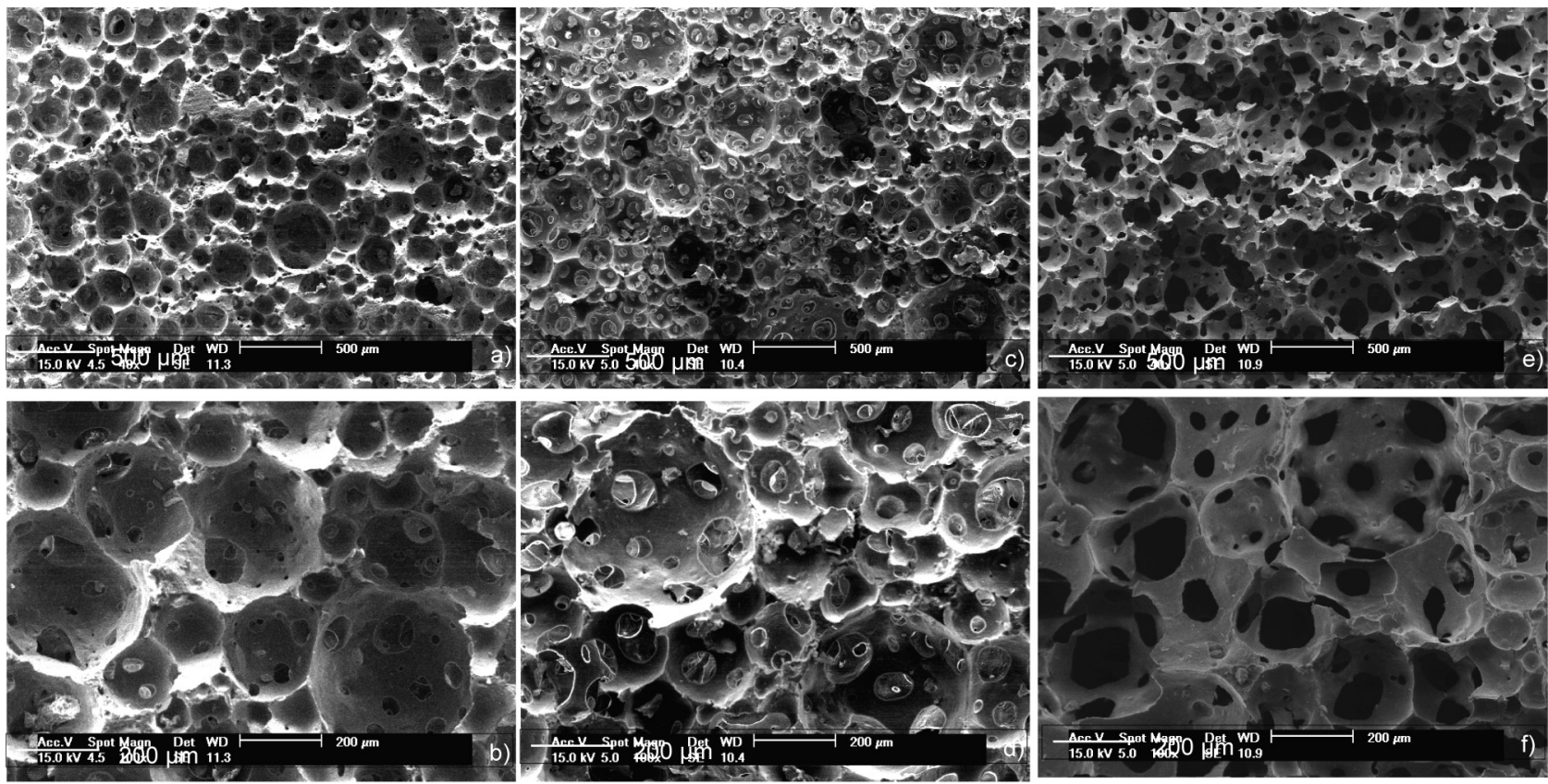

Figura 3: Micrografias das espumas de alumina obtidas com diferentes concentrações do agente espumante Fongraminox sem controle atmosférico: a, b) 0,4\% agente espumante; c, d) 0,5\% agente espumante; e, f) 0,6\% agente espumante.

[Figure 3: SEM micrographs of alumina foams obtained with different concentrations of foaming agent Fongraminox without atmospheric control: $a, b) 0.4 \%$ foaming agent; $c$, d) $0.5 \%$ foaming agent; $e, f$ ) $0.6 \%$ foaming agent.]
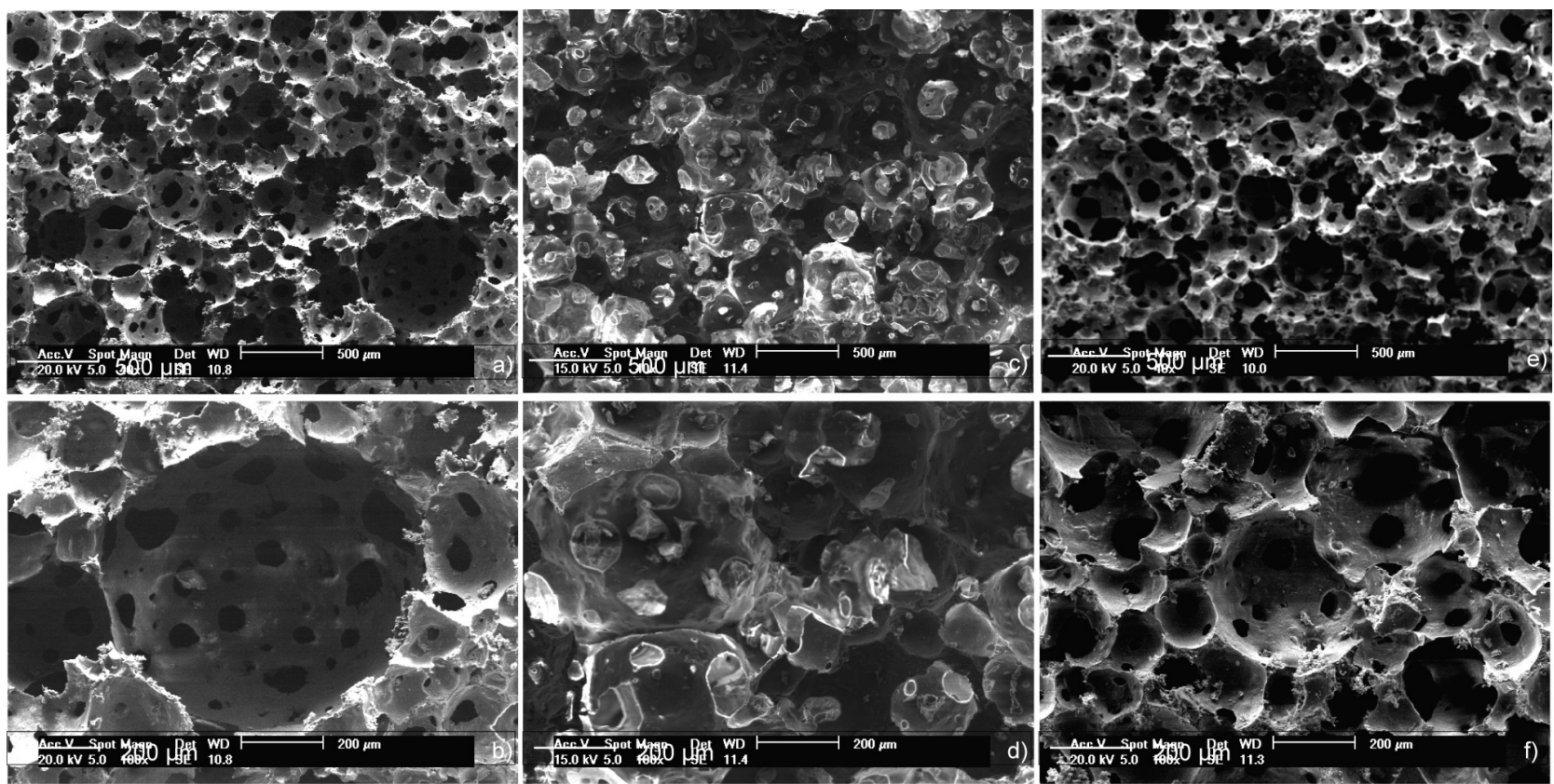

Figura 4: Micrografias das espumas de alumina obtidas com diferentes concentrações $(0,4,0,5$ e $0,6 \%)$ do agente espumante Lutensol sem controle atmosférico: a, b) 0,4\% agente espumante; c, d) 0,5\% agente espumante; e, f) 0,6\% agente espumante.

[Figure 4: SEM micrographs of alumina foams obtained with different concentration of foaming agent Lutensol without atmospheric control: $a$, b) $0.4 \%$ foaming agent; $c$, d) $0.5 \%$ foaming agent; $e, f) 0.6 \%$ foaming agent.]

numa estreita faixa, $81-85,5 \%$. Espumas com valores iguais de porosidade $(81 \%)$ foram conseguidos quando preparadas com $0,4 \%$ de agente espumante (Lutensol e Fongraminox). As espumas preparadas em atmosfera controlada apresentaram valores de porosidade mais elevados, $89-94,4 \%$ com Fongraminox e $85-94,4 \%$ com Lutensol. A diferença de valores de porosidade obtidos com e sem controle atmosférico está provavelmente associada a diferentes condições de processamento, tais como viscosidade da suspensão, tipo de monômero usado 
e temperatura, já que é pouco provável que alterações na composição da fase gasosa afetem a estabilidade da espuma.

As micrografias das espumas de alumina obtidas com diferentes concentrações $(0,4,0,5$ e $0,6 \%)$ do agente espumante Fongraminox sem controle atmosférico são mostradas na Fig. 3. Em todos as amostras observa-se uma macroestrutura homogênea, com poros aproximadamente esféricos, interconectados por janelas nas paredes das células, as quais são formadas nos pontos de contato entre bolhas vizinhas. Observa-se uma quantidade maior dessas janelas nas espumas de maior porosidade (Figs. 3d e 3f), ao mesmo tempo em que ocorre uma redução na espessura dos filamentos que constituem as paredes das células.

A Fig. 4 mostra as micrografias das espumas de alumina obtidas com diferentes concentrações $(0,4,0,5$ e $0,6 \%)$ do agente espumante Lutensol sem controle atmosférico.
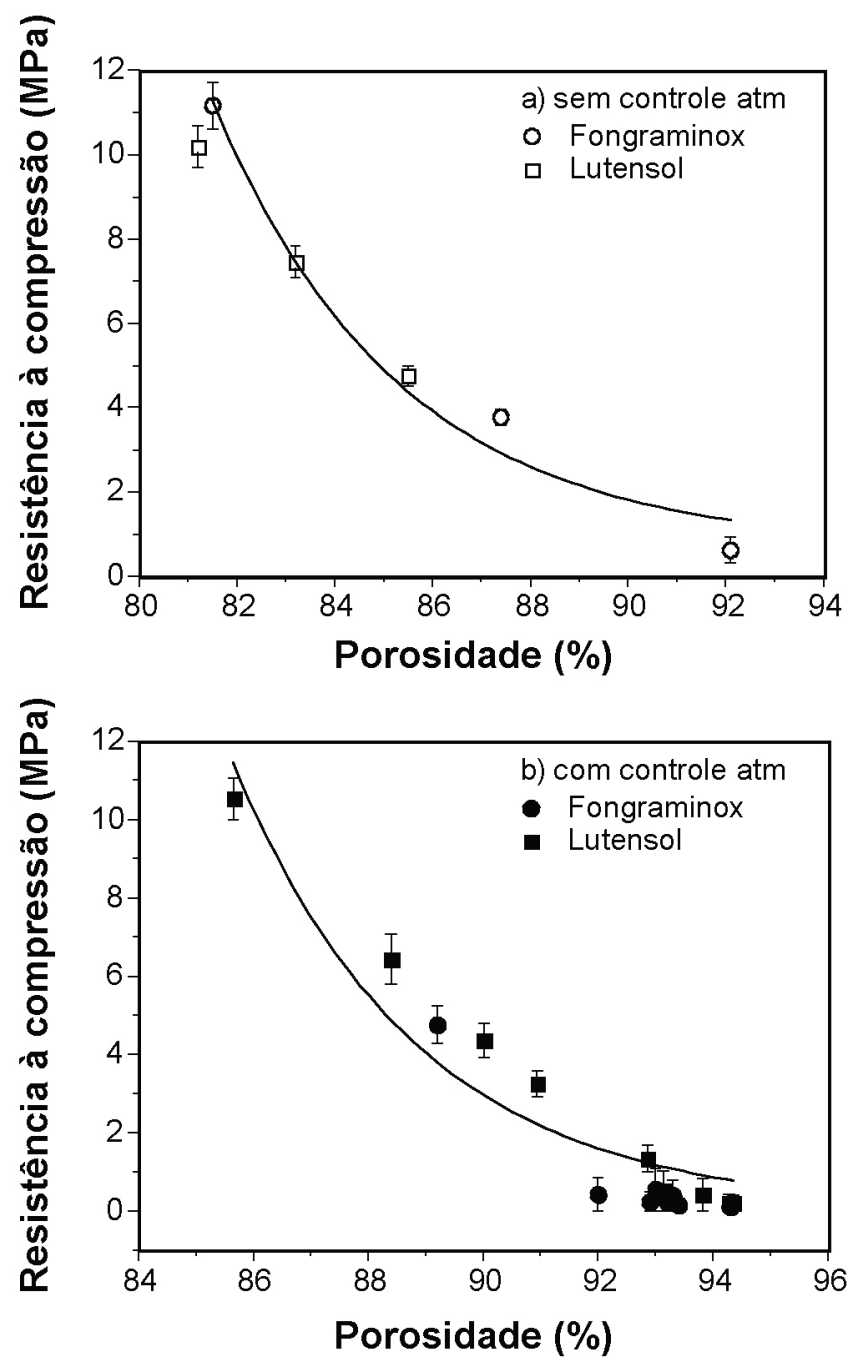

Figura 5: Resistência à compressão das espumas de alumina em função da porosidade: a) sem controle atmosférico e b) com controle atmosférico (adaptado da ref. 14).

[Figure 5: Compressive strength of alumina foams as a function of porosity: a) with atmospheric control and b) without atmospheric control (adapted of ref. 14).]
Novamente observa-se uma macroestrutura homogênea com poros aproximadamente esféricos e interconectados por janelas nas paredes das células.

Com base nas Figs. 3 e 4 observa-se que o tipo de agente espumante utilizado na produção das espumas cerâmicas não afeta a sua macroestrutura (morfologia e distribuição de tamanho de poros). Esta é apenas influenciada pela concentração de surfactante.

O comportamento mecânico das espumas de alumina é mostrado na Fig. 5. A resistência à compressão para as espumas obtidas sem controle atmosférico variou entre 0,6$11 \mathrm{MPa}$ (Fongraminox) e 4,5-10 MPa (Lutensol). Enquanto que para as espumas preparadas com controle atmosférico a resistência mecânica variou na faixa de $0,10-4,5 \mathrm{MPa}$ (Fongraminox) e 0,2-10,5 MPa (Lutensol). A diferença nos valores de resistência mecânica observados para amostras produzidas com diferentes surfactantes deve-se principalmente aos valores de porosidade obtidos em cada caso, já que a resistência mecânica diminui com o aumento da porosidade.

A Fig. 6 mostra a resistência à compressão das espumas de alumina em função da densidade relativa. Em ambos os casos, a resistência mecânica diminui com a redução da densidade relativa. Por meio de uma regressão exponencial sobre os conjuntos de pontos obtidos, é possível fazer uma comparação com o modelo de Gibson-Ashby (equação A). Para as espumas preparadas sem controle atmosférico, obtém-se um valor de $n$ igual a 2,5, enquanto que para as preparadas com o controle atmosférico o valor de $n$ foi de 2,3. Valor de $\mathrm{n}$ similar foi encontrado para espumas cerâmicas de alumina mulita $(n=2,2)$ [17]. A diferença no valor de $n$ pode ser explicada pelo fato do modelo de Gibson-Ashby basear-se no colapso catastrófico de células cúbicas homogêneas, sem defeitos em seus filamentos sólidos.

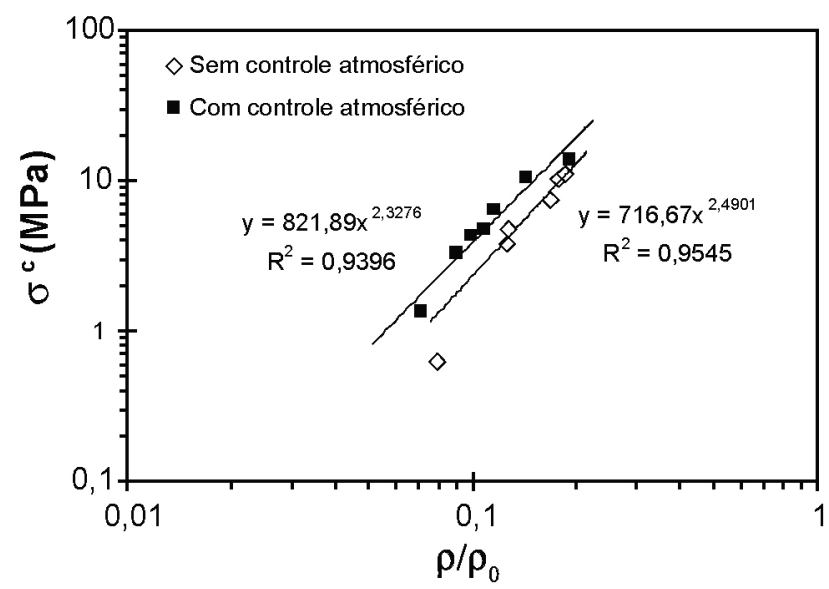

Figura 6: Resistência à compressão das espumas de alumina produzidas com ou sem controle atmosférico (adaptado da ref. 14) em função da densidade relativa.

[Figure 6: Compressive strength of alumina foams produced with and without atmospheric control (adapted of ref. 14) as a function of relative density.] 
No entanto, o valor de $n$, além de depender do modelo micromecânico usado, também depende da proporção de células abertas e fechadas presentes na estrutura celular, dos defeitos microestruturais existentes nos filamentos sólidos e do mecanismo de transferência de carga entre os constituintes do material [18]. Fatores esses que não são levados em consideração pelo modelo de GibsonAshby.

A Fig. 6 mostra também que, de um modo geral, as amostras produzidas sem controle atmosférico apresentaram uma resistência mecânica ligeiramente inferior à das amostras produzidas com controle atmosférico. De fato, o valor do produto $\sigma_{\mathrm{s}} \cdot \mathrm{C}_{1}$ (equação A) foi de $716,7 \mathrm{MPa}$ para amostras produzidas sem controle atmosférico e de $821,9 \mathrm{MPa}$ para amostras produzidas com controle atmosférico. Considerando que a constante $\mathrm{C}_{1}$ apresenta o mesmo valor em ambos os casos, essa diferença pode ser atribuída a uma menor resistência mecânica dos filamentos $\left(\sigma_{\mathrm{s}}\right)$ das amostras obtidas sem controle atmosférico.

\section{CONCLUSÕES}

Espumas de alumina foram produzidas com sucesso pela técnica gelcasting associada à aeração da suspensão cerâmica sem controle atmosférico. Para que a reação de polimerização não fosse inibida pela presença de oxigênio, quantidades elevadas de catalisador e iniciador foram utilizadas. O tipo de agente espumante utilizado (Fongraminox e Lutensol) não afetou a macroestrutura das espumas cerâmicas. Em ambos os casos, as espumas apresentaram poros geometricamente esféricos e interconectados. Com os dois tipos de agentes espumantes foi possível obter espumas com alta porosidade $(>80 \%)$. O comportamento mecânico das espumas de alumina pôde ser explicado pelo modelo de Gibson-Ashby, e sua resistência à compressão variou entre $0,6-10 \mathrm{MPa}$. Resultados semelhantes foram encontrados para as espumas obtidas com controle atmosférico.

\section{AGRADECIMENTOS}

Os autores agradecem ao CNPq pelo suporte financeiro.

\section{REFERÊNCIAS}

[1] J. Zeschky, F. Goetz-Neunhoeffer, J. Neubauer, S. H. Jason Lo, B. Kummer, M. Scheffler, P. Greil, Comp. Sc. Tech. 63 (2003) 2361.

[2] M. D. Leheigh, I. Nettleship, Mater. Res. Soc. Symp. Proc. 371 (1995) 315.

[3] S. Komarneni, L. Pach, R. Pidugu, Mater. Res. Soc. Symp. Proc. 371 (1995) 285.

[4] F. F. Lange, K. T. Miller, Adv. Ceram. Mater. 2, 4 (1987) 827.

[5] E. Sousa, C. B. Silveira, T. Fey, P. Greil, D. Hotza, A. P. N. Oliveira, Adv. Appl. Ceram. 104, 1 (2005) 22.

[6] W. M. Sigmund, N. S. BelL, L. Bergström, J. Am. Ceram. Soc. 83, 7 (2000) 1557.

[7] A. C. Young, O. O. Omatete, M. A. Janney, P. A. Menchhofer, J. Am. Ceram. Soc. 74, 3 (1991) 612.

[8] P. Sepulveda, Am. Ceram. Soc. Bull. 76, 10 (1997) 61.

[9] P. Sepulveda, J. G. P. Binner, J. Eur. Ceram. Soc. 19 (1999) 2059.

[10] M. A. Janney, O. O. Omatete, C. A. Walls, S. D. Nunn, R. J. Ogle, C. G. Westmoreland, J. Am. Ceram. Soc. 81, 3 (1998) 581.

[11] F. S. Ortega, P. Sepulveda, V. C. Pandolfelli, J. Eur. Ceram. Soc. 22 (2002) 1395.

[12] F. S. Ortega, F. A. O. Valenzuela, C. H. Scuracchio, V. C. Pandolfelli, J. Eur. Ceram. Soc. 23, 1 (2003) 75.

[13] L. J. Gibson, M. F. Ashby, Cellular solids: structure and properties, Cambridge University Press, $2^{\text {nd }}$ Ed., Cambridge, UK (1997).

[14] F. S. Ortega, J. A. Rodrigues, V. C. Pandolfelli, Am. Ceram. Soc. Bull. 83, 3 (2004) 9501.

[15] M. K. Gupta, R. Bansil, Polym. Prep. 22, 2 (1981) 375.

[16] T. M. Volkmer, Obtenção e caraterização de hidroxiapatita porosa pelo método de gelcasting de espumas para uso como implantes, Universidade Federal do Rio Grande do Sul (2006) p. 139.

[17] R. Brezny, D. J. Green, J. Am. Ceram. Soc. 76, 9 (1993) 2185.

[18] F. A. C. Oliveira, S. Dias, M. F. Vaz, J. C. Fernandes, J. Eur. Ceram. Soc. 26 (2006) 179.

(Rec. 03/03/2008, Ac. 16/05/2008) 